\title{
Development of Novel Therapies for Pulmonary Hypertension by Clinical Application of Basic Research
}

\author{
Kimio Satoh and Hiroaki Shimokawa
}

\begin{abstract}
Pulmonary hypertension is a lethal disease in which inflammation and oxidative stress are deeply involved in the disease state. We have recently screened novel pathogenic molecules and have performed drug discovery targeting those molecules. To confirm its clinical significance, we used patient-derived blood samples to evaluate the potential as a biomarker for diagnosis and prognosis. Finally, we conducted a high-throughput screening and found inhibitors for the pathogenic proteins. In this chapter, we would like to introduce the recent progress on the basic and clinical research focusing on the screening of pathogenic proteins in pulmonary hypertension.
\end{abstract}

\section{Keywords}

Pulmonary hypertension · Transnational research · Drug discovery $\cdot$ Biomarker Diagnosis

\subsection{Introduction}

Pulmonary arterial hypertension (PAH) is characterized by intimal/medial thickening and perivascular inflammation and fibrotic change. In addition to genetic backgrounds, many environmental factors as well as volume overload due to heart disease and inflammation due to collagen disease are involved in the development of PAH. The identification of pathogenic genes, which induce the abnormal characteristics of pulmonary artery smooth muscle cells (PASMCs), should be useful for

K. Satoh $(\bowtie) \cdot$ H. Shimokawa

Department of Cardiovascular Medicine, Tohoku University Graduate School of Medicine,

Sendai, Japan

e-mail: satoh-k@cardio.med.tohoku.ac.jp

(C) The Editor(s) (if applicable) and The Author(s) 2020

T. Nakanishi et al. (eds.), Molecular Mechanism of Congenital Heart Disease

and Pulmonary Hypertension, https://doi.org/10.1007/978-981-15-1185-1_16 
the development of novel therapies for PAH. The characteristics of PASMCs of patients with PAH (PAH-PASMCs) are different from those of healthy controls. In this chapter, we would like to summarize our recent progress on the basic and clinical research focusing on the screening of pathogenic proteins in $\mathrm{PAH}$.

\subsection{Endothelial Function in the Development of PAH}

Endothelial dysfunction triggers a variety of vascular disorders such as PAH. We have reported a protective role of the endogenous erythropoietin (Epo)/Epo receptor (EpoR) system against the development of $\mathrm{PH}$ [12]. This system also plays a crucial role in the functional recovery of ischemic heart [20] and ischemic lower limb [6], demonstrating the importance of endothelial function [4, 10]. Additionally, we found that pravastatin and metformin protect pulmonary endothelial function and ameliorate hypoxia-induced $\mathrm{PH}$ in animals $[8,11]$. AMP-activated protein kinase (AMPK) is a serine/threonine kinase that functions as an important energy sensor. AMPK has an anti-apoptotic effect in endothelial cells and a pro-apoptotic effect in vascular smooth muscle cells (VSMCs). Both endothelial nitric oxide (NO) production and NO-mediated signaling in VSMCs are targets of the AMPK. In endothelial cells, AMPK positively regulates NO production. In VSMCs, AMPK reduces secretion of growth factors that promote VSMC proliferation and vascular remodeling. Indeed, we have demonstrated that endothelial AMPK plays an important role in microvascular homeostasis in mice in vivo [2]. Additionally, AMPK activators (e.g., statins, metformin, and apelin) are protective against the development of PAH. We also demonstrated that endothelial AMPK plays protective roles against hypoxiainduced $\mathrm{PH}$ in mice [8, 11]. Circulating inflammatory cytokines downregulate AMPK and induce endothelial dysfunction in pulmonary circulation [9, 17]. Thus, endothelial AMPK as well as circulating inflammatory cytokines may be therapeutic targets for the treatment of PAH. Thus, AMPK is a key molecule at the crossroad of inflammation and pulmonary artery endothelial dysfunction in the pathogenesis of PAH.

\subsection{PASMCs in the Development of PAH}

Pulmonary vascular inflammation plays a crucial role for the development of hypoxia-induced PH [11, 12], for which Rho-kinase plays a crucial role $[1,3,16]$. Excessive and continuous activation of Rho-kinase promotes secretion of cyclophilin A (CyPA) from VSMCs and extracellular CyPA stimulates VSMC proliferation [13]. Additionally, extracellular CyPA induces endothelial cell adhesion molecule expression and endothelial apoptosis [7]. Basigin (Bsg) is an extracellular CyPA receptor. Bsg is also known as an essential receptor for Malaria, which disrupts NO metabolism and causes harmful endothelial activation, including the Rho/Rhokinase activation. Based on these backgrounds, we have demonstrated that CyPA and Bsg promote hypoxia-induced $\mathrm{PH}$ [15]. More importantly, plasma CyPA was 
significantly increased in patients with PAH and well correlated with the disease severity and long-term survival. Thus, extracellular CyPA and its signaling through Bsg are novel therapeutic targets for PAH. We have previously reported that statins and Rho-kinase inhibitors reduce CyPA secretion from VSMCs [14, 19]. Thus, inhibition of CyPA secretion by Rho-kinase inhibitors may have therapeutic efficacy in PAH. In addition, Bsg is strongly expressed in the pulmonary arteries of patients with PAH [15]. Thus, pharmacological agents that prevent the interaction of extracellular CyPA and vascular Bsg could be useful for the treatment of PAH. Indeed, our drug discovery research demonstrated that celastrol significantly inhibits CyPA and Bsg, inhibiting proliferation of PAH-PASMCs [18]. Here, we used PASMCs and high-throughput screening to identify novel agents to inhibit both CyPA and Bsg. PASMCs in the remodeled pulmonary arteries have special characteristics with pro-proliferative features. Importantly, celastrol suppressed CyPA and Bsg expressions in the heart and lung and ameliorated both heart failure (HF) and post-capillary $\mathrm{PH}$. Taken together, inhibiting both CyPA and Bsg may represent a novel therapeutic strategy for the treatment of HF patients with post-capillary $\mathrm{PH}$ [18]. As patients with $\mathrm{HF}$ and coexisting post-capillary $\mathrm{PH}$ show poor clinical outcomes, targeting both cardiac dysfunction and pulmonary vascular remodeling could be a novel concept for the treatment of HF.

\subsection{Selenoprotein P in the Development of PAH}

Since conventional pulmonary vasodilators have limited efficacy for the treatment of severe PAH, we have performed a series of screens and tried to find a novel therapeutic target. To identify a novel pathogenic protein, we performed microarray analyses using PAH-PASMCs and found 32-fold upregulation of selenoprotein $\mathrm{P}$ (SeP) as compared with control PASMCs [5]. Indeed, SeP is highly upregulated in the distal pulmonary arteries of PAH patients. SeP is a secreted protein mainly produced by hepatocytes, which contains 10 selenocysteine residues and transports selenium to maintain cellular metabolism. SeP is upregulated in the liver of patients with type 2 diabetes and downregulates the metabolic switch, AMPK. Additionally, single-nucleotide polymorphisms in the SEPPl gene have been reported to be associated with abdominal aortic aneurysm formation. Based on these backgrounds, we further demonstrated that SeP in PASMCs promotes cell proliferation through increased oxidative stress and mitochondrial dysfunction in an autocrine/paracrine manner [5]. Moreover, we demonstrated a pathogenic role of SeP in the development of hypoxia-induced PH in vivo. Since there is a limited efficacy in the treatment of patients with severe PAH, we performed a series of screening to find a novel therapeutic target. Finally, we identified that sanguinarine, an orally active small molecule, reduces SeP expression and PASMC proliferation and ameliorates $\mathrm{PH}$ in mice and rats. When we consider the pro-proliferative role of SeP in PAH-PASMCs, the anti-proliferative effect of sanguinarine in several kinds of cancer in vivo could be attributed to the suppression of SeP. Actually, sanguinarine administration to the animal models of $\mathrm{PH}$ revealed therapeutic effects on $\mathrm{PH}$ and $\mathrm{RV}$ failure without any 
adverse effects [5]. Moreover, serum levels of SeP were significantly elevated in $\mathrm{PAH}$ patients in whom higher serum levels of SeP predicted a poor outcome. Based on these results, serum levels of SeP can be used as a novel biomarker for PAH and are useful to evaluate the therapeutic effect of SeP inhibitors (companion diagnostics). Using a combination of SeP inhibitors and serum levels of SeP, we may find good candidates among PAH patients that can be used to demonstrate the effectiveness of this strategy.

\subsection{Conclusion}

In this chapter, we introduced our recent findings on Epo, AMPK, Rho-kinase, CyPA, Bsg, and SeP, all of which are substantially involved in the pathogenesis of PAH. Additionally, we have also mentioned as to the screening of inhibitors for those pathogenic proteins. By using the novel biomarkers and therapeutic agents, we will continue translational research for the early diagnosis and the development of fundamental therapy in $\mathrm{PAH}$ patients.

Acknowledgments This work was supported in part by the grants-in-aid for Scientific Research (15H02535, 15H04816, and 15K15046) from the Ministry of Education, Culture, Sports, Science and Technology, Tokyo, Japan; the Ministry of Health, Labour, and Welfare, Tokyo, Japan (10102895); and the Japan Agency for Medical Research and Development, Tokyo, Japan (15ak0101035h0001, 16ek0109176h0001, and 17ek0109227h0001).

\section{References}

1. Elias-Al-Mamun M, Satoh K, Tanaka S, et al. Combination therapy with fasudil and sildenafil ameliorates monocrotaline-induced pulmonary hypertension and survival in rats. Circ J. 2014;78:967-76.

2. Enkhjargal B, Godo S, Sawada A, et al. Endothelial AMP-activated protein kinase regulates blood pressure and coronary flow responses through hyperpolarization mechanism in mice. Arterioscler Thromb Vasc Biol. 2014;34:1505-13.

3. Ikeda S, Satoh K, Kikuchi N, et al. Crucial role of Rho-kinase in pressure overload-induced right ventricular hypertrophy and dysfunction in mice. Arterioscler Thromb Vasc Biol. 2014;34:1260-71.

4. Kagaya Y, Asaumi Y, Wang W, et al. Current perspectives on protective roles of erythropoietin in cardiovascular system: erythropoietin receptor as a novel therapeutic target. Tohoku J Exp Med. 2012;227:83-91.

5. Kikuchi N, Satoh K, Kurosawa R, et al. Selenoprotein p promotes the development of pulmonary arterial hypertension. Circulation. 2018;138:600-23.

6. Nakano M, Satoh K, Fukumoto Y, et al. Important role of erythropoietin receptor to promote VEGF expression and angiogenesis in peripheral ischemia in mice. Circ Res. 2007;100:662-9.

7. Nigro P, Satoh K, O'dell MR, et al. Cyclophilin A is an inflammatory mediator that promotes atherosclerosis in apolipoprotein E-deficient mice. J Exp Med. 2011;208:53-66.

8. Omura J, Satoh K, Kikuchi N, et al. Protective roles of endothelial AMP-activated protein kinase against hypoxia-induced pulmonary hypertension in mice. Circ Res. 2016;119:197-209.

9. Rabinovitch M, Guignabert $C$, Humbert $M$, et al. Inflammation and immunity in the pathogenesis of pulmonary arterial hypertension. Circ Res. 2014;115:165-75. 
10. Satoh K, Fukumoto Y, Nakano M, et al. Emergence of the erythropoietin/erythropoietin receptor system as a novel cardiovascular therapeutic target. J Cardiovasc Pharmacol. 2011;58:570-4.

11. Satoh K, Fukumoto Y, Nakano M, et al. Statin ameliorates hypoxia-induced pulmonary hypertension associated with down-regulated stromal cell-derived factor-1. Cardiovasc Res. 2009;81:226-34.

12. Satoh K, Kagaya Y, Nakano M, et al. Important role of endogenous erythropoietin system in recruitment of endothelial progenitor cells in hypoxia-induced pulmonary hypertension in mice. Circulation. 2006;113:1442-50.

13. Satoh K, Matoba T, Suzuki J, et al. Cyclophilin A mediates vascular remodeling by promoting inflammation and vascular smooth muscle cell proliferation. Circulation. 2008;117:3088-98.

14. Satoh K, Nigro P, Matoba T, et al. Cyclophilin A enhances vascular oxidative stress and the development of angiotensin II-induced aortic aneurysms. Nat Med. 2009;15:649-56.

15. Satoh K, Satoh T, Kikuchi N, et al. Basigin mediates pulmonary hypertension by promoting inflammation and vascular smooth muscle cell proliferation. Circ Res. 2014;115:738-50.

16. Shimizu T, Fukumoto Y, Tanaka S, et al. Crucial role of ROCK2 in vascular smooth muscle cells for hypoxia-induced pulmonary hypertension in mice. Arterioscler Thromb Vasc Biol. 2013;33:2780-91.

17. Soon E, Holmes AM, Treacy CM, et al. Elevated levels of inflammatory cytokines predict survival in idiopathic and familial pulmonary arterial hypertension. Circulation. 2010;122:920-7.

18. Sunamura S, Satoh K, Kurosawa R, et al. Different roles of myocardial ROCK1 and ROCK2 in cardiac dysfunction and postcapillary pulmonary hypertension in mice. Proc Natl Acad Sci U S A. 2018;115:E7129-38.

19. Suzuki J, Jin ZG, Meoli DF, et al. Cyclophilin A is secreted by a vesicular pathway in vascular smooth muscle cells. Circ Res. 2006;98:811-7.

20. Tada H, Kagaya Y, Takeda M, et al. Endogenous erythropoietin system in non-hematopoietic lineage cells plays a protective role in myocardial ischemia/reperfusion. Cardiovasc Res. 2006;71:466-77.

Open Access This chapter is licensed under the terms of the Creative Commons Attribution 4.0 International License (http://creativecommons.org/licenses/by/4.0/), which permits use, sharing, adaptation, distribution and reproduction in any medium or format, as long as you give appropriate credit to the original author(s) and the source, provide a link to the Creative Commons license and indicate if changes were made.

The images or other third party material in this chapter are included in the chapter's Creative Commons license, unless indicated otherwise in a credit line to the material. If material is not included in the chapter's Creative Commons license and your intended use is not permitted by statutory regulation or exceeds the permitted use, you will need to obtain permission directly from the copyright holder.

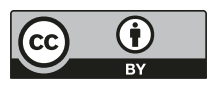

